\title{
MILITARY AVIONICS TWENTY YEARS IN THE FUTURE
}

\author{
D.Reed Morgan \\ Avionics Directorate \\ Wright Laboratory \\ Wright-Patterson Air Force Base, Ohio
}

\begin{abstract}
This paper explores the generic structure of avionics beyond the Pave Pace system architecture. Projected constraints, opportunities and trends in several pervasive system building blocks which should be mature by this time frame are discussed and an example of the form this architecture might take is offered. In developing these projections, the author interviewed several technology experts in Industry and Government to help in forecasting the future of military avionics. General conclusions are: (1) costdriven sensor solutions will drive the avionics designs for most military avionics applications in order to meet demanding situation awareness goals, (2) the most pervasive and highest-leveraged building blocks used to build this advanced system will be commerciallyavailable data and signal processors along with advanced $\mathrm{A} / \mathrm{D}$ converters and digital and $\mathrm{RF}$ photonics, (3) a continued increase in time sharing and physical integration at every level of the avionics architecture is projected, including multifunction EO and $\mathrm{RF}$ apertures, $\mathrm{RF}$ support electronics and a highly integrated digital system, (4) distributed high-speed photonic switches will permeate the architecture to achieve a unified interconnect network across RF, IF, data and signal processing modules built from families of printed wiring boards, (5) advances in packaging and microelectronics will result in the need for liquid immersion cooling and 3-dimensional stacking of RF, digital and photonic circuitry to support integrated apertures, digital receivers and data and signal processors to accomodate the needed speed and functional integration, (6) the digital boundary will move closer to the apertures to enable IF digital receivers for electronic warfare and radar applications (digital receivers up through L-band will have occurred during the Pave Pace era) and will extend the use of photonics beyond the digital arena to where photonically-controlled beam steering for phased arrays, RF signal distribution and hetereodyning will be possible.
\end{abstract}

\section{INTRODUCTION}

Predicting the characteristics of the next military avionics system beyond Pave Pace, which is not yet demonstrated, is obviously difficult because many assumptions must be made about the future direction of military priorities, budgetary constraints and the degree of success researchers will have in maturing several key technologies. This paper assumes the drive for low cost avionics will continue and that the 20 year system will evoive from the Pave Pace system introduced around the 2007-10 time frame. Therefore, one of the tasks at hand is to identify pervasive, highly-leveraged building block technologies, unavailable to the Pave Pace system that can further reduce avionics costs. For the 20 year design, these technologies should be in the early stages of prototype device development and significant funding interest must be shown by DoD departments such as the Advanced Research Projects Agency (ARPA).

\section{FUTURE AVIONICS NEEDS}

In the author's opinion, the greatest challenge facing future military avionics is to contain costs while providing the sensor and processing capability that will enable situation awareness and survivability against mobile threats in a system configuration which is ultra-reliable and supportable for austere base operation. The flyaway costs of fighter avionics (measured as a percentage of weapon system flyaway cost and exclusive of software costs) has increased steadily since the 1960 s from about $12 \%$ for the F-4 to almost $40 \%$ today. Looking at cost, weight, volume, failure rates and electrical power requirements for advanced fighter avionics, Pave Pace studies showed that almost $60 \%$ of the values of these parameters could be attributed to RF sensors while about $20 \%$ of "the problem" is attributed to processing and its associated networks (the remaining $20 \%$ being the electronic portion of stores management, vehicle management and controls and displays). 
The conclusion to be drawn is that avionic systems twenty years in the future must exhibit substantially reduced costs for both sensors and processing, while at the same time it should provide a very robust situation awareness capability. An apparent conflict emerges since situation awareness is normally achieved with improved sensor and processing capability.

In order to achieve the needed performance improvements in sensors and processing at lower costs, a new sensor architecture is needed that departs substantially from current-day systems. As will be discussed later, the first step in this new architecture has been initiated by the Integrated Sensor System portion of the Pave Pace avionics design.

\section{EVOLUTION OF AVIONICS SYSTEMS}

New avionics systems evolve from their predecessors, using new technologies to overcome network bottlenecks (resulting from the availability of advanced processing) which, in succeeding digital device generations, will eventually cause another new system to be needed. If we are to understand where avionics is going, we need to understand the cyclic forces at work. Figure 1 shows how military avionics has responded to the drive for improved capability over time through an exponential increase in data and signal processing capability [1]. This Figure reflects the pervasive nature of digital computers in driving avionics designs. For example, digital processing got underway in the 1960 s by implementing air data, navigation and weapon delivery algorithms in blackbox data processors: digital signal processing followed during the 1970 s, complex automation, information fusion algorithms and real-time sensor processing (e.g., SAR imaging) became feasible in the 1980/90 time frame and in the future, digital $R F$ and IF receivers will become a reality. Many of the processing requirements are impressive: for example, pulse compression for the ground moving target indication mode for advanced radars will require about 1500 MOPS. The evolution of avionics can be viewed as a steady movement of the $A / D$ boundary closer to the skin of the aircraft through several generational steps. The system processing projections shown in Figure 1 indicate that the 20 year avionic system will require about 2 billion instructions per second for data processing and about 50-100 BOPS of signal processing.

Four major avionic system generations have emerged since the $1960 \mathrm{~s}$. The reader should be aware that these generational designations are somewhat arbitrary since there is an evolutionary blurring between them. They are: 1) Independent systems (analog and/or digital black box computers with hard-wired interconnects; this type of system was on the F-111 and A-7), 2) Federated (digital data processor black boxes with one Megabit/sec MIL STD 1553 interconnects; this generation is typical of aircraft flying today), 3) Integrated Modular Digital systems (modular data and signal processors in racks); this generation will appear on the F-22 aircraft after the turn of the century), 4) Integrated Modular Avionics (modular data, signal and RF avionics in racks interconnected by 2 Gigabits/sec interconnects; this generation is called Pave Pace and should appear around the year 20072010).

One can observe a dominant trend across these avionic generations: functional and physical integration has increased with time to enable improved situation awareness (e.g., automation, fusion of data), improved testability (reduce maintenance personnel and groundbased test equipment) or to reduce cost and weight by reducing the amount of hardware needed (invariably at the expense of more complex software).

During each avionics generation, the following cyclic process occurs: initially, dramatic speed and functionality improvements at the device level are made available as microcircuit foundries have reduced feature size; next, dense, ruggedized packaging approaches are then developed, leading to increased physical and functional integration. Computer cost and weight is reduced not only from lower-cost devices, but the low volume of the computer makes it practical to share power supplies, backplanes, racks and operating systems (with improved testability, fault tolerance and data fusion made much easier at the system level and new algorithms are now exploited within the computing system; operating system software however is made much more complex); eventually a data bottleneck is reached as the integrated processor is unable to support the faster data stream from the sensors and to the displays. Although both device speed and network speed have grown exponentially over the last three decades, network speeds have not kept pace.

Increased processor speed and functionality is the basic goal of a multi-billion dollar, highly competive commercial industry which thrives on improvements. Further, equally impressive strides have been made in packaging these advanced devices into the small volumes needed for computer speed and ruggedization for avionics. More functions, previously implemented in physically separate black boxes or modules can now be included within the new packaged module or integrated rack. In turn, the system network must 
increase in speed to provide the needed $\mathrm{I} / \mathrm{O}$, although fewer separate networks are needed. Eventually, functional integration reaches a point where streaming data across "Iong distances" between sensors (aircraft skin), the processing complex (at easily accessible locations) and displays (cockpit) dominate. Continued increases in network speeds then will occur at a slower rate since system functional computing consolidation having occurred. Network speeds are now such that photonics becomes practical because of the weight and complexity of high-power circuits required to drive high speed digital signals over several meters of (high resistance) wire or coax. Within the computing complex, signalling speeds higher than the network level is needed within and between PWBs. A speed level is eventually reached where the electrical backplane is too slow (again due to resistance) and direct MCM-MCM 3-D interconnects between PWBs are needed. These very short interconnects will likely continue to be electrical for the foreseeable future because electrical-optical conversion costs and accompanying speed delays make photonics impractical.

The Pave Pillar architecture was developed during the 1980 s to enable processing and network assets to be configured in a more highly integrated manner. Functions performed in large black boxes under the Federated system are accomplished in small $(6 \times 6 \times 6$ inch) modules through the exploitation of VLSI technology, advanced multi-chip packaging and liquid flow through cooling. In-rack co-habitation of modular signal processors executing radar, $\mathrm{CNI}$, and electronic warfare functions is accomplished across a unified backplane in the common integrated processor (CIP). Sensor data can now be easily fused and combined with graphic processors to provide the intelligent display of information. The common design allows the sharing of built-in test hardware and software to enable flight-line removal of the modules without the use of the avionics intermediate shop. Further, the integrated design eases reconfigurability of assets to dramatically increase system availability.

However, many of these benefits came as a result of moving the signal processing assets from the sensor support hardware to the CIP, resulting in a need to provide a very high data rate path. In effect, the backplane traffic between the pre-processor and the signal processor for a Federated System must now occur across a physical network to interconnect the pre-processor with the CIP. Similarly, graphics processors located previously at the display heads must be network-connected between the CIP and the display. The current network implementation is several $400 \mathrm{Megabit} / \mathrm{sec}$ photonic-based, point-to-point links. Further, a $50 \mathrm{Megabit} / \mathrm{sec}$ photonic bus is used to interconnect the racks and mass memories. Inside the rack, a new hierarchical network structure was needed to handle high speed back plane traffic. These electrical parallel networks include a 32 bit, 800 Megabit/sec switch to route streaming sensor data into signal processors modules (through global memories) and two buses, one for system control and low speed data passing (PI bus at $400 \mathrm{Megabits} / \mathrm{sec}$ ) and one for test and maintenance functions.

Although this design represents the most-advanced avionic system yet built, the Pave Pace Program was initiated in 1989 to look at downstream enhancements which would be made possible by previously unavailable technologies. The resulting design uses technology available around the year 2000 .

\section{The Pave Pave Architecture}

Figure 2 shows the top-level features of the Pave Pace Architecture. Note the evolutionary nature of this design relative to Pave Pillar where the common digital modular family concept has been extended into $\mathrm{RF}$ support electronics to form a low cost, light weight Integrated Sensor System (ISS). Through exploitation of MMIC circuitry, advanced RF packaging and resource sharing, half-cost, half-weight integrated $R F$ systems will be built by mixing an matching modules. Figure 2 shows that multi-function, shared RF apertures for radar and part of the EW spectrum and L-band CNI avionics and multi-function EO/IR sensors (shared windows, optics, dewars, etc). Further, a "unified protocol" based fault tolerant networkswitched, high speed (e.g., about 2 Gigabit/sec) laserbased photonic network is employed to interconnect digitized sensor data to functional clusters of modules within the CIP and to interconnect clusters to clusters, racks to racks and racks to displays.

Several interesting trends can be observed with this architecture. First, the trend for continued functional and physical integration and the use of common modules continues unabated in order to reduce cost and weight. Second, higher speed laser-based interconnects are now needed to accomodate the streaming data brought on by increased processor speed and added functionality concentrated in the CIP. This Very High Speed Optical Network (VHSON) photonic switched network (Figure 3) also replaces the backplane-based electrical switched network used by the previous architecture (also eliminating some costly electrical to photonic energy conversion circuitry). Note that the telephone-like switched network has 
replaced buses and has been elevated from the rack to the system level (sensors, CIPS, displays) to support streaming data and to achieve fault tolerance. This architecture also reflects the dominance that signal and graphics processors will play in the future. It is anticipated that commercial-based data and signal processors packaged with Multi-Chip Modules and Chip on Board technology will be used. Typical digital modules are expected to consume 100-200 watts (despite 1-2 volt devices) and will require liquid flow through cooling and on-module electrical power conditioning to permit operation at higher backplane voltages (e.g., 50 volts) to reduce the connector problems caused by high amperage (if a 5 volt backplane continues to be used). It is projected that the modular data and signal processors will run at over 1000 MIPS and well over 1000 MFLOPS respectively. The form, fit and interface specifications of this design will be provided through an Open System Architecture to maximize competition and drive down costs. Pave Pace studies show that the emerging commercial digital technology will reduce the processing module count by a factor of ten.

\section{TWENTY YEAR ARCHITECTURE}

We are now in a position to forecast the generic characteristics of the 20 year system. We must first look at the building-block technologies that will be available, along with new packaging techniques either required or available for further exploitation, then determine the system architecture implications of this new capability in reducing the cost of Pave Pace Avionics.

Figure 4 show projected processor speeds for commercial data and signal processors[1]. Extapolating to the 2015 time frame, we see that even if these trends are assumed to taper off substantially, devices should (conservatively) run at about 100 million MIPS and 10 million MOPS (these projections allow for a reduction of performance growth as practical limits of device feature size, clock rate and reduced voltages might be reached). The system implications of these digital device performance improvements will be profound. The extent that we can practically use this technology in military avionics will be determined by our ability to provide adequate network data rates, ruggedized packaging and adequate cooling. Conservatively, a 0.2-.5 TERAOP (peak) parallel-processor-based computer with over 1 Gigabyte memory (memory devices are expected to be well over 1 Gigabit by this time) in a (double-width) SEM-E size module should be practical. And if history is to be our guide, we will find several uses for it!
Hybrid and monolithic waters, including mixedsignalling (e.g., analog/digital, digital/photonic) devices will be used to achieve the speeds projected. These continued impressive strides will have profound effects on the way in which military avionics will be packaged, cooled and interconnected and, as discussed below, will allow a new sensor architecture to evolve.

Since devices will generate several tens of watts, operating at $1 \mathrm{Ghz}$ (device level) clock rates, they must be physically close. Liquid immersion cooling (already in practical use for supercomputers and high power RF power sources) will be mandatory to cool commercial computing devices to 70 degrees $C$ (or even 125 degree C). Further, 3-D stacking of PWBs, using compliant electrical interconnects (avoiding the backplane) to permit direct (vertical) communication between devices will be needed to maintain required system $\mathrm{L} / \mathrm{O}$, clocking and computation speeds. In effect, a parallel processing network will be built on a single PWB with memory either on the same PWB or in immediate proximity through $3-D$ interconnects. Due to high clock speeds, many of the packaging approaches currently being used in RF avionics will likely be required (e.g., low temperature co-fired ceramic, RF isolation). Hughes Research Laboratory, AT\&T and others have already shown the viability of 3-D packaging using current-day digital signalling speeds and Westinghouse has developed high density monolithic packaging for RF that has solderless connections. Using packaging approaches such as chip on board, hybrid wafers and even monolithic wafers for memories, a high-performance, complete computing system can be built within one 3-D stack (ie, $\mathrm{I} / \mathrm{O}$, signal conditioning, pre-processing, signal processing, data processing and memory) by mixing and matching a family of PWBs within a line replaceable module. The new CIP will consist of a few of these very, very powerful computers. Advanced high speed algorithms performing such functions as ultra-high resolution real-time SAR processing and improved automatic target recognition algorithms will exploit the new computing technology. Unmanned Airborne Vehicles will also have the added capability of complete automation.

However, the power of this computing capability will force fundamental changes to occur in several areas. First, we will not be able to continue adding complex, diverse algorithms to the computing module/CIP at the same rate as in the past; we cannot afford the software costs; despite the belief that software production efficiencies will improve, the author does not believe these efficiencies will off-set the hardware "cost savings". Therefore, the number of these highly 
advanced modules in the CIP will not be large. (Note: we are starting to resemble the uniprocessor architecture of the 1960s, with each "PWB" being thousands of times more capable).

Second, the same digital technology will be used to build digital receivers at the IF level. A family of digital IF receiver modules is envisoned. The ISS Open System Architecture has been designed to accept digital IF receiver upgrades up to the primary IF (about $2.25 \mathrm{GHz}$ ) without requiring modification. Using future commercial signal processing devices (not currently available), several benefits should result, including lower cost and improved reliability (fewer custom analog mixer, oscillator and filter circuits), reduced calibration problems resulting from highperformance analog circuits and improved BIT. Significant progress in digital receivers is being made. For example, the technology goal of a current ARPAsponsored Millennium Program is to demonstrate a packaged digital receiver capable of 3 Gigasamples/per sec with 8 bit resolution (adequate for channelized receivers). Hughes Research has built a 4 bit/8Gigasample/sec A/D device. Pave Pace technology forecast studies project the availability of A/D converters capable of supporting general IF digital processing by the year 2005. Certain radar applications requiring large spur free dynamic ranges (e.g., 130dB) may be the exception however and second digital IF sampling may be required. The general form the 3-D packaged implementation of a Digital IF Processor will take involves vertically interconnected PWBs for signal conditioning, A/D conversion, digital filtering (e.,g., FIR filters), Fast Fourier Transformation and pre-processing functions. Note the pre-processing function (previously separate modules) has been integrated into the 3-D stack. As with the digital processing modules in the advanced CIP (integrated data, signal and graphics processing), advanced RF packaging, signal isolation and immersion cooling will be required for digital receivers.

Photonic technology used in the RF domain will also play a dominant role in shaping the 20 year architecture. Pave Pace technology forecast studies project the cost of single mode fiber and high speed (e.g., laser diodes) to fall steadily during this decade due to commercial usage. For example, a $10 \mathrm{GHz}$ laser diode is projected to cost $\$ 100$ by the year 2000 . The implications of this technology are immense. RF photonic signals will be routed to and from apertures; IF up/down conversion hardware and phased-arrays will be controlled through true time delays across large frequency bands and optical heterodyning of RF to IF signalling will occur. Much lighter weight, lower cost and lower noise RF sensor avionics will result, along with lower parts count. Currently, a Boeing/Hughes Research Lab team is developing an RF optical modulator (externally modulated) with a $16 \times 16$ lithium niobate switch to generate, route and control $\mathrm{RF}$ signals up to $18 \mathrm{Ghz}$ under an ARPA-sponsored effort. Optical heterodyning is just beginning to emerge from the laboratory.

Figure 5 shows the author's current best estimate of the 20 year architecture. Multifunction RF apertures are assumed to be commonplace with tile-based arrays being used. High temperature superconductors have begun to appear in selective applications such as RF frequency sources. Comparing this diagram with Figure 2 (Pave Pace), one can see the A/D interface has move up to the IF photonic switch. Analog photonics have (mainly) replaced the microwave down-conversion hardware and coax cables between this hardware and the apertures. The optically switched system network remains although possibly network speeds of "only" about 5 Gigabits/sec may need needed. The CIP consists of fewer modules, now interconnected by the photonic switch (instead of functional cluster of modules for Pave Pace). Most controversial is the integration of stores, vehicle and utilities management processing functions with general sensor and data processing in order to further save cost and weight by sharing of switch, backplane, rack, etc assets. The author believes that since advanced software and hardware isolation techniques are now available to satisfy multi-level security concerns brought on by integrated avionics, safetycritical issues resulting from integration will eventually be satisfied as well.

\section{Acknowledgements}

The author is indebted to several individuals who have provided insight into the future of military avionics. Contributors include: M. Hamilton/Boeing and Dr J. Schroeder/Harris (Photonics), Dr S. Oxford/Hughes (A/D converters), D. Sharpin/Avionics Directorate, and R. Bobb, C. Eppers/Solid State Directorate of Wright Laboratory (A/D converters, digital receivers), P. Ryan/Avionics Directorate (High Temperature Superconductors) and K. Leahy/Westinghouse (RF packaging).

\section{References}

1. Data provided by McDonnell-Douglas Aerospace Company, St Louis, Missouri. 


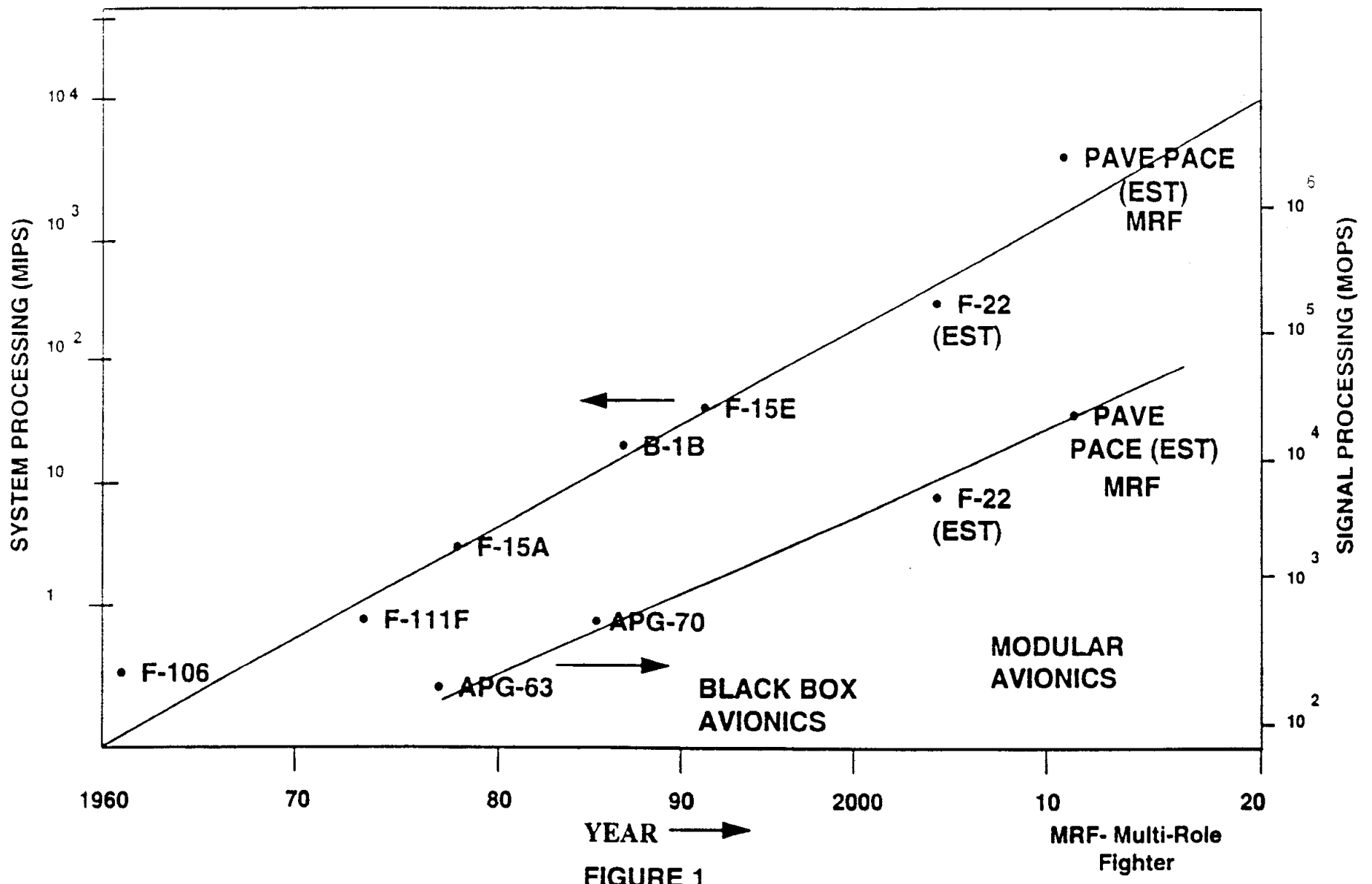

EVOLUTION OF DIGITAL AVIONICS PROCESSING

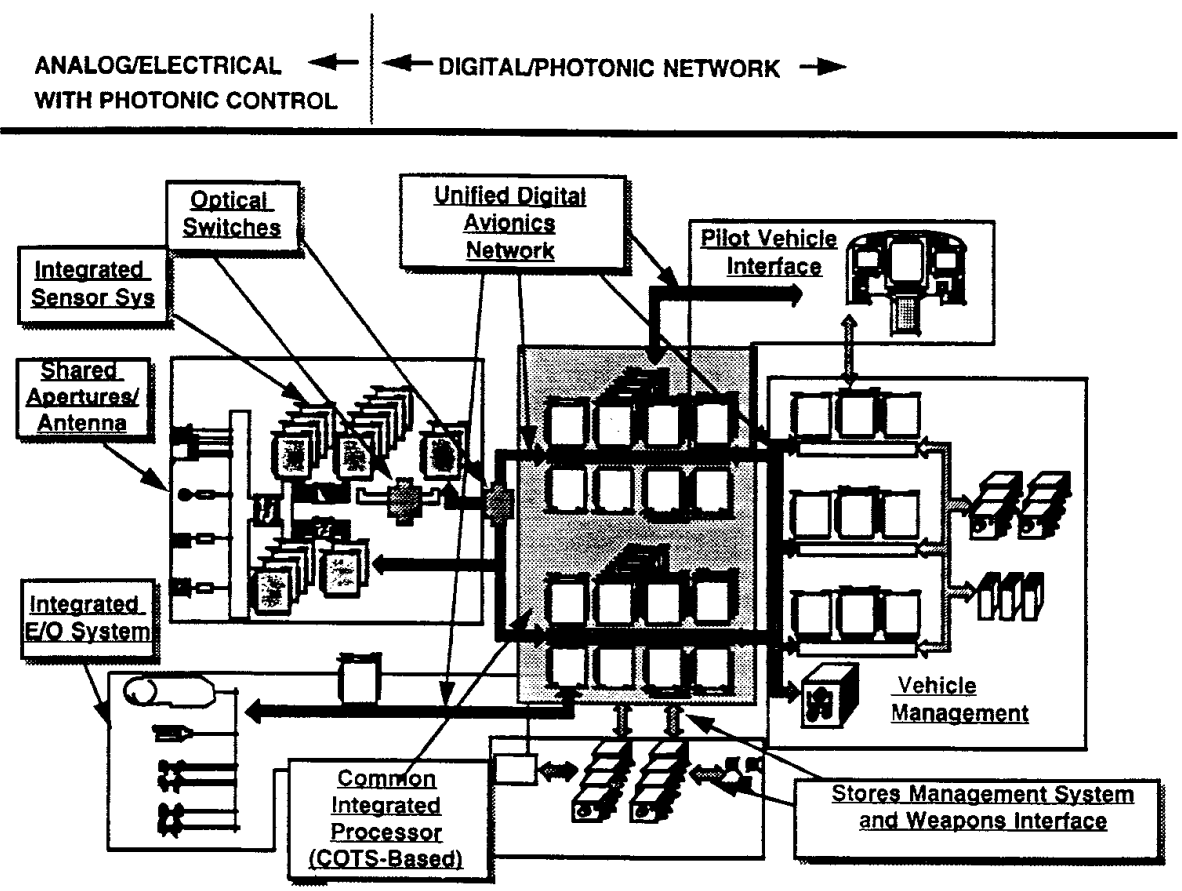

FIGURE 2

PAVE PACE ARCHITECTURE 


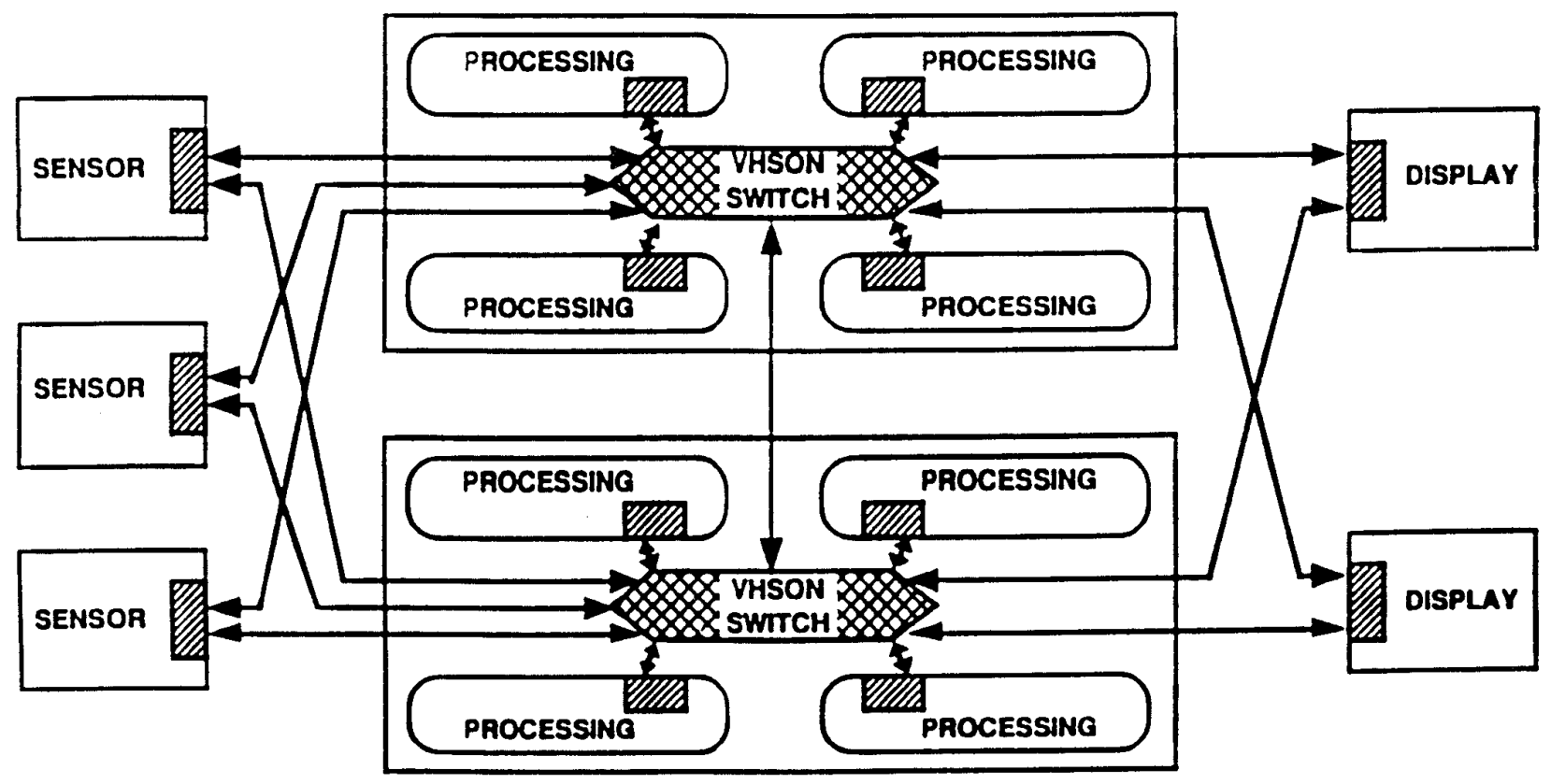

WIIA VHSON INTERFACE CIRCUTT (VIC)

FIGURE 3

OPTICALLY SWITCHED CIRCUITS

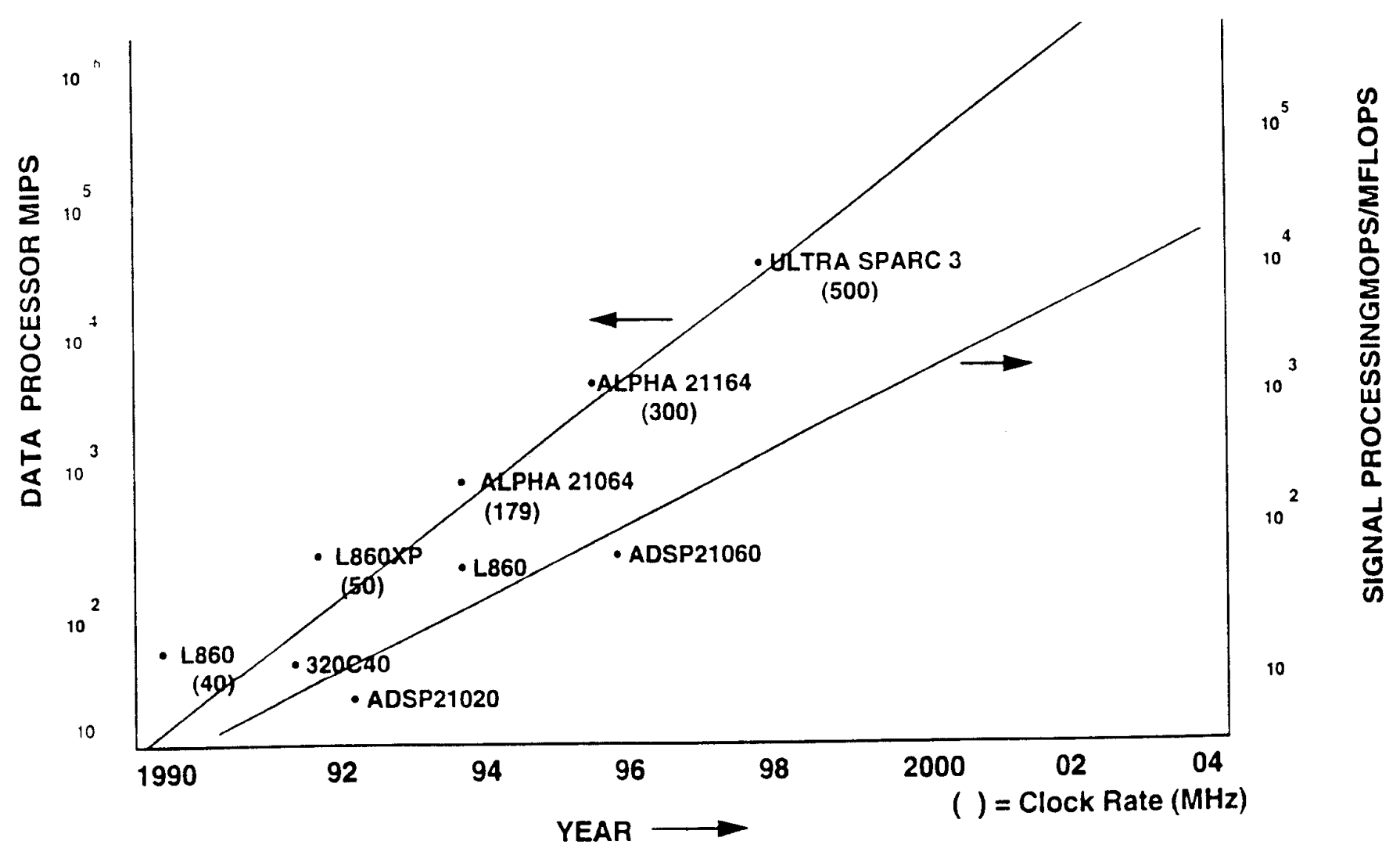

FIGURE 4

COMMERCIAL MICRO ELECTRONICS DEVICE PERFORMANCE 


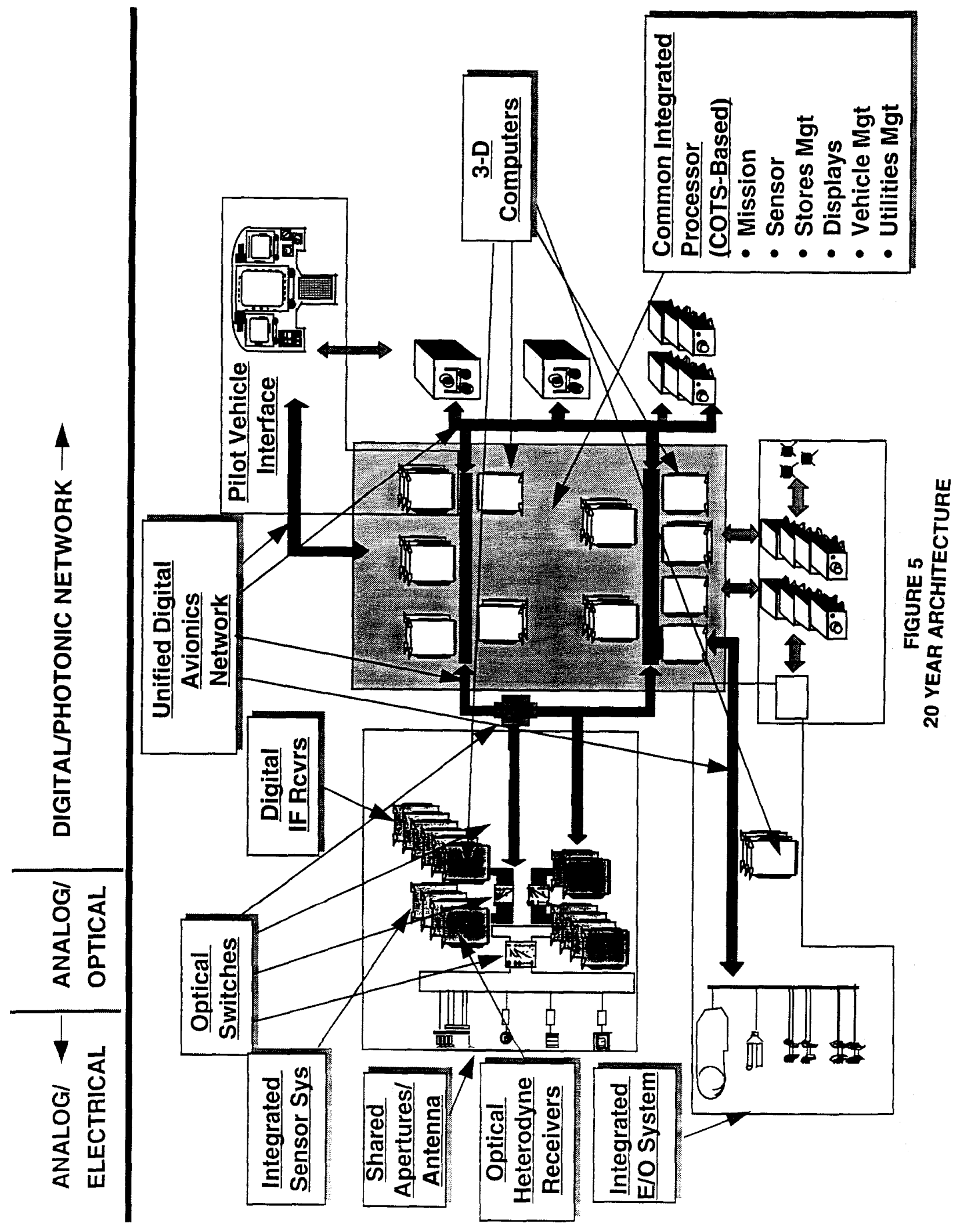

